\title{
RESIDÊNCIA MULTIPROFISSIONAL EM SAÚDE DA FAMÍLIA COMO CONDUTORA DE EDUCAÇÃO PERMANENTE NA ATENÇÃO BÁSICA
}

MULTIPROFESSIONAL RESIDENCE IN FAMILY HEALTH AS A CONDUCTOR OF PERMANENT EDUCATION IN BASIC ATTENTION

RESIDENCIA MULTIPROFESIONAL EN SALUD DE LA FAMILIA COMO CONDUCTORA DE EDUCACIÓN PERMANENTE EN LA ATENCIÓN

BÁSICA

Francisco Timbó de Paiva Neto ${ }^{1}$

Antonio Cleilson Nobre Bandeira 2

Palavras-chave:

Educação Permanente;

Tecnologias em Saúde; Equipe

Multiprofissional.

Keywords:

Permanent Education; Health Technologies; Multiprofessional

Team.

Palabras clave:

Educación Permanente;

Tecnologías en Salud; Equipo

Multiprofesional

Submetido:

04/11/2019

Aprovado:

$11 / 12 / 2019$

Autor(a) para

Correspondência:

Francisco Timbó de Paiva Neto

Universidade Federal de Santa Catarina

Departamento de Educação Física-DEF/CDS/UFSC

Campus Universitário, Trindade

88040-900, Florianópolis, SC

E-mail:timbonetto@gmail.com

\section{RESUMO}

O texto objetiva relatar os processos de Educação Permanente em Saúde (EPS) realizado pela Residência Multiprofissional em Saúde da Família no município de Florianópolis, Santa Catarina e promover reflexão sobre a percepção do conceito de Educação Permanente em Saúde pelos profissionais da Atenção Básica. As EPS aconteceram nos espaços de planejamento dos Centros de Saúde, a fim de aprimorar e organizar os encontros, além de assegurar a integralidade e significado do mesmo no processo de trabalho dos profissionais. Frente aos discursos dos sujeitos podemos perceber que as suas percepções sobre Educação Permanente estão muito próximas das que são definidas pela PNEPS como Educação continuada, mesmo após a condução de momentos conduzidos pelos residentes. Conclui-se que a inserção de residentes multiprofissionais nos serviços de saúde, integrando-se aos processos de trabalho do Grupo Tutorial/EPS, tem como um dos desígnios potencializar métodos na melhoria da saúde em parceria com os trabalhadores nos seus territórios.

\footnotetext{
1. Profissional de Educação Física, Doutorando em Educação Física pela Universidade Federal de Santa Catarina, Florianópolis, Santa Catarina. E-mail: timbonetto@gmail.com ORCID: https://orcid.org/0000-0002-5477-3645 2. Profissional de Educação Física, Especialista Residente em Saúde da Família pela Universidade Estadual Vale do Acaraú. Sobral, Ceará. E-mail: clenobre@hotmail.com ORCID: https://orcid.org/0000-0002-0314-1146
} 


\section{ABSTRACT}

The purpose of this article is to report the processes of Permanent Health Education (EPS) realized by the Multiprofessional Residency in Family Health at Florianópolis city, Santa Catarina and promote reflection over the perception of the concept of Permanent Health Education by Primary Care professionals. The EPS occurred in the circles of coexistence spaces of the Health Centers in order to improve and organize the meetings, besides ensuring the integrality and meaning of the same in the work process of the professionals. In front of the subjects' discourses we can see that their perceptions about Permanent Education are very close to those defined by the PNEPS as Continuing Education, even after conducting moments realized by the residents. It is concluded that the insertion of multiprofessional residents in health services, integrating in the work processes of the GT / EPS, has as one of the main intentions to potentialize methods in improving health in partnership with workers in their territories.

\section{RESUMEN}

El texto objetiva relatar los procesos de Educación Permanente en Salud (EPS) realizado por la Residencia Multiprofesional en Salud de la Familia en el municipio de Florianópolis, Santa Catarina y promover reflexión sobre la percepción del concepto de Educación Permanente en Salud por los profesionales de la Atención Básica. Las EPS se realizaron en los espacios de rueda de los Centros de Salud, a fin de perfeccionar y organizar los encuentros, además de asegurar la integralidad y significado del mismo en el proceso de trabajo de los profesionales. Frente a los discursos de los sujetos podemos percibir que sus percepciones sobre Educación Permanente están muy próximas a las que son definidas por la PNEPS como Educación continuada, incluso después de la conducción de momentos conducidos por los residentes. Se concluye que la inserción de residentes multiprofesionales en los servicios de salud, integrando a los procesos de trabajo del GT / EPS, tiene como uno de los planes potenciar métodos en la mejora de la salud en asociación con los trabajadores en sus territorios.

\section{INTRODUÇÃ 0}

Em 2004, por meio da Portaria No 198/2004, o Ministério da Saúde instituiu a Política Nacional de Educação Permanente em Saúde (PNEPS) como estratégia do Sistema Único de Saúde (SUS) para a formação e o desenvolvimento de trabalhadores para o setor. Esta política define a Educação Permanente em Saúde (EPS) como um instrumento para mudanças e transformações dos serviços de saúde. As transformações sociais e educacionais têm repercussões nos modos de produzir, nos diferentes campos do saber e de produção de bens e serviços ${ }^{1}$.

Com efeito, as relações entre educação e trabalho em saúde assumem posições, estratégias e significados importantes, procurando vencer a dicotomia entre pensar e fazer, integrando habilidades teóricas e práticas. A EPS pretende incorporar o ensinar e o aprender ao cotidiano das organizações e do trabalho ${ }^{1,2}$. Portanto, a EPS busca constantemente a aprendizagem, possibilitando processos e desenvolvimentos de profissionais de saúde para estarem comprometidos com a assistência, conscientes e responsáveis, inseridos no processo de trabalho e no território, conhecedores do sistema em que atuam, com suas políticas e ações ${ }^{1}$.
Assim, a EPS apresenta-se como proposta de ação estratégica capaz de contribuir para a transformação dos processos formativos, das práticas pedagógicas, de atenção à saúde e para a organização dos serviços, empreendendo um trabalho articulado entre 0 sistema de saúde, em suas várias esferas da gestão, e as instituições formadoras. Nesse sentido, a educação permanente por ser um modelo inovador de buscar transformar uma realidade, deve ultrapassar os campos do saber focal dos profissionais e buscar o ensino-aprendizagem amplamente, a partir do seu dia-a-dia com a comunidade e, assim, dar continuidade ao processo ensino-serviço.

0 sistema de EPS vem sendo compreendido no mesmo momento que um conjunto de alternativas educacionais, articuladas que visam o desenvolvimento de ações voltadas para aprendizagem significativa no processo de trabalho. A EPS prevê a construção de conhecimentos nos serviços de saúde, instituindo-se a aprendizagem no trabalho, onde o aprender e o ensinar se incorporam ao cotidiano das organizações e dos serviços ${ }^{3,4}$. EPS propõe uma nova forma de produzir conhecimento e de pensar a educação e o trabalho.

É necessário introduzir a EPS como estratégia fundamental das práticas de formação, atenção, 
gestão, formação de políticas e controle social no setor saúde. Assim, estabelecendo ações intersetoriais com o setor educação, garantindo os processos de mudanças necessárias na graduação, nas residências em saúde, na pós-graduação, para que haja uma relação entre direitos da população e da universalização das ações e serviços de saúde ${ }^{5}$.

Sobre as ferramentas de apoio às Equipes de Saúde da Família (ESFs) de Florianópolis destacamse a Residência Multiprofissional em Saúde da Família (RMSF) que pode ser compreendida, além de um processo de formação, como uma estratégia de mudança da formação dos trabalhadores da saúde, com construção interdisciplinar, trabalho em equipe, educação permanente e de reorientação das práticas para atuação diferenciada no SUS. Nesse campo de atuação coletiva, os programas de residências multiprofissionais ganham espaços nos quais ocorrem as trocas de saberes.

Reconhece-se que diante do atual contexto da saúde pública no Brasil, a estratégia da residência multiprofissional enquanto ferramenta de reflexão das ações vem se reafirmando como imprescindível mecanismo de problematização de mudanças no modelo de atenção em saúde que supere o foco das ações na doença e na execução de procedimentos ${ }^{6-8}$. A partir desta reflexão, o sistema de saúde de Florianópolis, pautado na pedagogia dialógica problematizadora que estabelecem espaços cotidianos de reflexão coletiva sobre os processos de trabalho, aposta na implementação de momentos mensais de EPS dentro do espaço da reunião de todos os Centros de Saúde (CS) do município, onde é planejado, executado e avaliado pela RMSF, e desta forma, os momentos possam agregar conhecimento no cotidiano de trabalho dos profissionais das ESFs.

Este estudo buscou refletir as contribuições da Residência Multiprofissional em Saúde da Família nos espaços de Educação Permanente em Saúde para discutir questões discernentes ao processo de trabalho, fomentando a reflexão crítica e proativa dos trabalhadores por meio de discursos dos profissionais de saúde participantes.

\section{METODOLOGIA}

Trata-se de um estudo exploratório-descritivo com abordagem qualitativa. A pesquisa exploratóriadescritiva tem como objetivo principal a descrição das características de determinadas populações ou fenômenos, e o estabelecimento de relações entre

\author{
... residência \\ multiprofissional (...) \\ vem se reafirmando \\ como imprescindivel \\ mecanismo de \\ problematização de \\ mudanças...
}

as variáveis ${ }^{11}$. A pesquisa qualitativa apresentase como uma abordagem interpretativa do mundo, onde seus pesquisadores buscam estudar as coisas em seus cenários naturais, tentando compreender os fenômenos em termos dos significados que as pessoas a eles atribuem ${ }^{12}$.

Neste tipo de abordagem, o fenômeno humano estudado é entendido como parte da realidade social, pois este não se difere só por agir, mas por pensar sobre o que faz, e interpretar suas ações dentro e a partir da realidade vivida e partilhada com seus semelhantes ${ }^{9,10}$.

A coleta ocorreu em Florianópolis, nos meses outubro e novembro 2017, em seis Centros de Saúde (CS) da sede do município com a participação de 164 profissionais da ESF de Florianópolis. Para a construção do corpus da pesquisa, utilizou-se o roteiro semiestruturado a partir do qual os sujeitos tiveram a possibilidade de discorrer sobre o tema abordado em cada momento de EPS. 0 projeto de pesquisa foi submetido e aprovado em seus aspectos éticos e metodológicos pelo Comitê de Ética em Pesquisa da Universidade Federal de Santa Catarina, e a Comissão Científica da Secretaria de Saúde de Florianópolis, de acordo com as normas que regulamentam a pesquisa em seres humanos, do Conselho Nacional de Saúde, Ministério da Saúde, Resolução n 466, de 12 de dezembro de 2012, obtendo aprovação sob o Parecer No 1.893.360/2017.

$0 \mathrm{~s}$ dados foram tratados mediante Análise de Conteúdo, um conjunto de técnicas de análise das comunicações, visando obter por procedimentos sistemáticos descrição do conteúdo das mensagens, indicadores que permitam a inferência de conhecimentos relativos às condições de produção/ recepção (variáveis inferidas) destas mensagens ${ }^{13}$.

Foram operacionalizados seguindo as três etapas da análise temática: a pré-análise, exploração do material, tratamento dos resultados obtidos 
e interpretação. A fase de pré-análise, que se subdividiu em leitura flutuante e preparação do material, consistiu na organização dos dados, sistematizando as ideias iniciais, por meio da visualização individual de cada diálogo, onde se destacou os aspectos considerados relevantes. Após a ordenação dos diálogos por meio da transcrição dos conteúdos foram feitas exaustivas leituras dos textos, organizando os relatos de modo a possibilitar o mapeamento das falas, agrupando-as por similaridade de ideias, assinalando as que tinham o mesmo significado ou semelhança, identificando as unidades significativas das falas a fim de que pudessem ser compreendidas e conscientemente exploradas ${ }^{13}$. Esta forma de organização permitiu visualizar as seguintes temáticas: atualizações de conhecimentos, aprendizado permanente e processo formativo no trabalho apresentadas a seguir. A experiência permanece em andamento até os dias atuais. Definiu-se também uma avaliação da experiência, em espaço de encontro administrativo da unidade, a cada seis meses de acordo com a necessidade dos participantes.

Desse modo, após pactuar em espaços de cogestão (reunião de gerentes das unidades), definiu-se que os profissionais das equipes de Residência em Saúde da Família seriam, a partir de então, atores que planejam, executam e participam dos momentos de EPS na perspectiva de apoiar o Grupo de Trabalho de Educação Permanente em Saúde (GT/EPS) do Sistema de Saúde de Florianópolis, em relação a aprimorar e organizar os encontros, além de assegurar a integralidade e significado do mesmo no processo de trabalho dos participantes.

\section{RESULTADOS E DISCUSSÃO}

0 estudo é resultante da inserção de uma equipe multidisciplinar de residentes em Saúde da Família, após vivências teórico-práticas nos espaços de planejamento de CS, onde entende-se que a EPS é um método educativo que coloca o cotidiano do trabalho ou da formação em saúde em análise. Sendo um processo necessário, pois a graduação é insuficiente para compreender as complexidades e desafios encontrados nos territórios.

Fomentada pela RMSF, a EPS possibilita o diálogo com a realidade do serviço, capaz de perceber o contexto e transformá-lo, estando diretamente relacionada com a prática. 0s residentes são subsidiados e preparados para estes momentos

\section{... a EPS possibilita \\ o diálogo \\ com a realidade \\ do serviço...}

de acordo com suas vivências teóricas e estudos dirigidos. Na RMSF, são vários os espaços que proporcionam EPS, como ciclos (exposição teórica), encontros de categoria, estudos multiprofissionais, vivências nos equipamentos da rede, participação em Encontros de Residências e Fóruns.

No território, a EPS ocorre em diferentes ocasiões de forma transversal, como em encontros das Unidades de Saúde, compartilhamento de casos, intervisitas, intergrupos, construção de Projeto Terapêutico Singular, mas para que os resultados sejam positivos é necessário conhecer as demandas do território, sua real necessidade. Percebe-se a importância da residência como estratégia de transformar a formação para o Sistema Único de Saúde (SUS), possibilitando a percepção do que necessita ser melhorado, e com a teoria e discussão fomenta as possibilidades de transformação da realidade por meio da efetivando a proposta de EPS.

A residência promove espaços de educação permanente capazes de ressignificar os processos de trabalho em saúde, melhorar as condutas profissionais e faz com que se sinta valorizado, uma vez que a EPS emerge de desafios e necessidades dos mesmos. 0s temas versam sobre diversos âmbitos: trabalho em equipe; saúde mental; promoção da saúde; educação em saúde; questão social; acolhimento, respeitando as demandas dos trabalhadores. Esses momentos de encontro entre ESFs, GT/EPS e residentes mostram-se efetivos na construção coletiva entre os atores envolvidos e permitiu às equipes olharem para seu fazer em saúde de modo que o processo de aprendizagem fosse permeado pelo protagonismo, contribuindo para o diálogo como forma de potencializar processos para melhoria do fazer cotidianamente.

0 s encontros dialógicos permitem inferir que a inserção de residentes multiprofissionais conduz a aprendizagem significativa para o próprio residente, quanto em qualificação do trabalhador de saúde, inclusive nas questões que dizem respeito ao processo de trabalho das equipes de ESF.

A mudança na atenção à saúde é uma necessidade 
imprescindível e desafiadora e tem na Educação Permanente em Saúde uma estratégia pujante para sua concretização. A residência tem a proposta de romper com o modelo de saúde biologicista e biomédico que gera atendimentos fragmentados e descontínuos; desvaloriza o saber dos outros profissionais, descaracterizando a construção de uma assistência multiprofissional com o cuidado holístico. Durante esse tempo de vivencia destacase a dificuldade em superar essas práticas devido ao processo de trabalho rígido por parte dos profissionais e gestores, o qual é mais pautado no cumprimento de metas do que em práticas que vislumbrem o atendimento integral, pela fragilidade da rede socioassistencial, pela desvalorização dos trabalhadores que desencadeia desmotivação e adoecimento.

Contudo, apesar dos entraves postos são muitas as possibilidades de avançar na perspectiva do atendimento integral, de contribuir com o processo de implementação do SUS e de organização da sociedade civil: processo de formação para preceptores e tutores, o adentro dos residentes nos diversos serviços da rede de saúde, a interlocução com outras residências multiprofissionais e médica e o perfil político dos residentes. A residência é uma experiência enriquecedora para o processo de formação político dos profissionais, bem como para o perfil dos mesmos.

A mesma por diferentes arranjos organizativos formadores de ações e serviços de diferentes configurações tecnológicas e missões assistências formada por equipes multiprofissionais que se articulam de forma complementar tendo como base o território ${ }^{14}$.

As percepções dos profissionais sobre os momentos de EPS foram organizadas em subcategorias que emergiram dos depoimentos dos sujeitos da

A mudança na atenção à saúde (...) tem na Educação Permenente em Saúde uma estratégia pujante para sua concretização... pesquisa: Atualização de Conhecimentos; Formação contínua que norteia o processo de trabalho. Como Atualização de Conhecimentos consideram a Educação Permanente uma ferramenta que consegue tirar dúvidas, atualizar, renovar e acrescentar seus conhecimentos, como pode ser constatado nos discursos:

“A Educação Permanente é uma excelente ferramenta para atualizar nossos conhecimentos, tirar dúvidas, 4 para facilitar o nosso trabalho" (Profissional 1).

"Para os profissionais de saúde é muito bom, pois estão sempre atualizando os conhecimentos, renovando, não se fica desatualizado das coisas. Eu gosto muito de Educação Permanente" (Profissional 3).

"Só veio para contribuir, acrescentar conhecimento, renovar e melhorar. Através da EP aprendemos a seguir os protocolos, trabalhar com mais segurança, estar atualizados, tirar dúvidas, a pessoa tem base, tem segurança para aplicar na sua prática" (Profissional 16).

Este entendimento evidencia que a educação permanente leva o profissional à competência, ao conhecimento e à atualização, que são componentes necessários para garantir a sobrevivência, tanto do profissional quanto da própria profissão. Podemos observar com os depoimentos que a percepção sobre Educação Permanente em Saúde foca-se em atualizações, novos conhecimentos, aprendizagem nos serviços. De acordo com os relatos, percebemos que as práticas de Educação Continuada são entendidas pela maioria dos profissionais como Educação Permanente.

Compreendemos que esse equívoco pode ter suas raízes no contexto o qual estamos inseridos, quando se atribui a qualquer atividade pedagógica relacionada aos serviços, o conceito de Educação Permanente. 0s sujeitos a conceituarem a EPS como atualizações, aquisição de conhecimentos, afirmam que ao vivenciarem esta experiência se sentem seguros para desenvolverem melhor as suas ações, como podemos observar nos depoimentos:

“Só veio para contribuir, acrescentar conhecimento, renovar, só veio para 
melhorar. Através da EPS usando protocolos, trabalhar com mais segurança, estar atualizados, tirar dúvidas, a pessoa tem base, tem segurança daquilo que aprendeu para aplicar na sua prática" (Profissional $7)$.

“Então eu vejo a Educação Permanente como um instrumento bastante favorável para o aprendizado do enfermeiro e consequentemente para uma boa realização do seu trabalho" (Profissional 23).

Ao analisarmos essas percepções, buscamos na literatura as afirmações de Davini ${ }^{15}$, que conceitua educação continuada como o conjunto de experiências subsequentes à formação inicial, que permitem ao trabalhador manter, aumentar ou melhorar sua competência, para que esta seja compatível com o desenvolvimento de suas responsabilidades, caracterizando, assim, a competência como atributo individual.

Entretanto, a abordagem da educação permanente pauta-se a partir do conhecimento emergido das situações as quais envolvem os profissionais no seu cotidiano de trabalho, razão pela qual a torna importante para a transformação do SUS e para o desenvolvimento dos profissionais inseridos neste. Nas instituições de saúde há uma tendência em se acreditar que o domínio do conhecimento científico é fator primordial para a qualidade dos serviços. Muitas vezes, os profissionais realizam cursos de atualização, treinamentos e capacitações com a expectativa de transformar sua prática.

Nesse contexto, predomina mais uma vez “[...] o valor do atributo conhecimento em detrimento do exercício de análise das condições concretas do processo de trabalho, que aponta a necessidade de intervenções múltiplas e criativas" ${ }^{16}$.

\section{...conhecimento$$
\text { emergido das }
$$$$
\text { situações as }
$$$$
\text { quais envolvem os }
$$$$
\text { profissionais no }
$$$$
\text { seu cotidiano de }
$$$$
\text { trabalho... }
$$

No que se refere à formação contínua que norteia o processo de trabalho, consideraram a EPS como um processo formativo capaz de dar continuidade à aprendizagem da graduação e que esta pode nortear para melhor organização dos serviços, conforme 0 que observamos nas falas abaixo:

“A EPS significa uma continuação da faculdade, é quando você recebe conteúdos programáticos e estruturados para melhorar o seu trabalho no território." (Profissional 8).

“A EPS serve para clarear tudo aquilo que a gente já viu na universidade há muito tempo, tanto dentro dos programas, da organização dos serviços, metodologias desenvolvidas, para que a gente possa planejar as ações na ESF." (Profissional 17).

“A educação permanente é uma bússola, um norte, mas se esta bússola não é bem gerida, a gente se perde no meio do caminho. A organização do serviço se deu pela educação permanente. A EPS é de grande valia. Dá certo quando queremos fazer." (Profissional 19).

Reportando-nos a literatura sobre definições e conceitos da EPS, podemos identificar na PNPS, que a educação continuada tem como pressuposto pedagógico o conhecimento para definir as práticas; como objetivo, a atualização de conhecimentos específicos; percebe como público alvo os profissionais em suas especificidades, de acordo com os conhecimentos a serem trabalhados; a operacionalização é descendente, acontece a partir de uma leitura geral dos problemas, identificamse temas e conteúdo a serem trabalhados com os profissionais, geralmente sob o formato de cursos.

As atividades educativas são pontuais e fragmentadas, sendo construídas de maneira desarticulada em relação à gestão, à organização do sistema e ao controle social ${ }^{15,17-18}$. Enquanto que a educação permanente em saúde segue os pressupostos pedagógicos apresentados pela OPAS/ OMS, ainda na década de 80 , pois parte das práticas definidas por múltiplos fatores, conhecimentos, valores, relações de poder, organização do trabalho, entre outros, a aprendizagem significativa requer que se trabalhe com elementos que "façam sentido" 
para os sujeitos sociais envolvidos e os coloquem em posição ativa de propor e reordenar as práticas. 0 objetivo principal é a transformação real das práticas, o público alvo, as equipes de atenção e de gestão multiprofissional e interdisciplinar em qualquer esfera do sistema. As atividades educativas são construídas de maneira ascendente, a partir da análise coletiva dos processos de trabalho, levando em conta as necessidades específicas de profissionais e equipes de saúde, identificam-se os "nós" críticos que devem ser enfrentados na atenção ou na gestão, - que possibilita a construção de estratégias contextualizadas que promovam o diálogo entre as políticas gerais e a singularidade dos lugares e das pessoas e conceitos ${ }^{5,15,18}$.

Portanto, frente aos discursos dos sujeitos podemos perceber que as suas percepções sobre Educação Permanente estão muito próximas das que são definidas pela PNEPS como Educação continuada, mesmo após a condução de momentos conduzidos pelos residentes.

\section{CONCLUSÃO}

Apesar da distinção entre as terminologias EPS, Continuada e em Serviço, todas têm caráter de continuidade do processo educativo, porém se fundamentam em diferentes princípios metodológicos. A EPS, além da capacitação técnica é também um compromisso pessoal a ser conquistado com as mudanças de atitudes decorrentes das experiências vividas, por meio da relação com os outros, com o meio, com o trabalho, buscando a transformação pessoal, profissional e social. Parte da aprendizagem significativa para que os sujeitos sociais envolvidos se coloque em posição ativa de propor e reordenar as práticas

Portanto, conclui-se que a inserção de residentes multiprofissionais nos serviços de saúde, integrandose aos processos de trabalho do GT/EPS, tem como um dos desígnios potencializar métodos na melhoria da saúde em parceria com os trabalhadores nos seus territórios. Além da discussão da estratégia de EPS apoiada por outros atores, são necessários estudos que verifiquem a repercussão das destes espaços no cotidiano laboral desses trabalhadores, conduzidos pelos profissionais que facilitam os momentos de educação permanente.

Percebe-se que a educação permanente representa uma estratégia que possibilita o estabelecimento de vínculo entre as redes de atenção em saúde,

\section{A EPS, além da capacitação técnica é também um compromisso pessoal...}

profissionais e comunidade promovendo assim a continuidade do cuidado e aumentando por meio da corresponsabilização da atenção a capacidade de resolutividade dos problemas de saúde mais comuns de forma a produzir um maior impacto na situação de saúde dentro de um determinado território.

\section{CONTRIBUIÇÃO DOS AUTORES}

Francisco Timbó de Paiva Neto contribuiu na concepção do projeto, coleta, análise e interpretação dos dados, redação e revisão crítica do artigo e aprovação da versão final a ser publicada. Antonio Cleilson Nobre Bandeira colaborou na coleta, análise e interpretação dos dados, redação e revisão crítica do artigo e aprovação da versão final.

\section{REFERÊNCIAS}

1. Balbino $A C$, Bezerra MM, Freitas CASL, Albuquerque IMN, Dias MAS, Pinto VPT. Educação permanente com os auxiliares de enfermagem da Estratégia Saúde da Família em Sobral, Ceará. Trab. Educ. Saúde [Internet]. 2010 [cited 2019 Mai 21];8(2):249-66. Available from: http://www.scielo.br/pdf/tes/v8n2/ a05v8n2.pdf

2. Ceccim RB, Feuerwerker LCM. Mudança na graduação das profissões de saúde sob o eixo da integralidade. Cadernos de Saúde Pública [Internet]. 2004 [cited 2019 Jul 18];20(5):1400-10. Available from: http://www.scielo.br/pdf/csp/v20n5/36.pdf

3. Barth OB, Aires M, Santos JLG, Ramos, FRS. Educação Permanente em Saúde: concepções e práticas de enfermeiros de unidades básicas de saúde. Revista Eletrônica de Enfermagem [Internet]. 2014 [cited 2019 Ago 10];16(3):604-11. Available from: https://www.revistas.ufg.br/fen/article/ view/22020/17542

4. Ministério da Saúde. Departamento de Gestão da Educação na Saúde. Política Nacional de Educação Permanente em Saúde: o que se tem produzido para o seu fortalecimento? [documento on the internet]. 2018 [cited 2019 0ut 20]. Available from: http://bvsms.saude.gov.br/bvs/publicacoes/ politica nacional educacao permanente saude fortalecimento.pdf 
5. Ceccim RB. Educação Permanente em Saúde: desafio ambicioso e necessário. Interface Comunic, Saúde, Educ [Internet]. 2005 [cited 2018 Dez 22];9(16):161-77. Available from: http://www. escoladesaude.pr.gov.br/arquivos/File/textos\%20 eps/educacaopermanente.pdf

6. Nascimento DDG, Oliveira MAC. Competências Profissionais e o Processo de Formação na Residência Multiprofissional em Saúde da Família. Saúde e Sociedade [Internet]. 2010 [cited 2019 Set 13];19(4):814-27. Available from: http://www. scielo.br/pdf/sausoc/v19n4/09.pdf

7. Santa Catarina (Estado). A Política Municipal de Educação Permanente em Florianópolis- SC. [document on the internet]. 2011 [cited 2019 Ago 16]. Available from: http://www.pmf.sc.gov. br/arquivos/arquivos/pdf/23 $01 \quad 2018 \quad 14.06 .29$. c0a18df455385ff8a5f538ca9f55ae71.pdf

8. Brum LM. A pedagogia da roda como dispositivo de educação permanente em enfermagem e a construção da integralidade do cuidado no contexto hospitalar [Dissertação] [Internet]. Porto Alegre. Universidade Federal do Rio Grande do Sul; 2009. 133 p. [cited 2019 Abr 02]. Available from: https://www.lume. ufrgs.br/handle/10183/18669

9. Dyniewicz AM. Metodologia da pesquisa em saúde para iniciantes. 2. ed. São Caetano do Sul: Difusão; 2009.

10. Minayo MCS. 0 desafio do conhecimento: pesquisa qualitativa em saúde. São Paulo: Hucitec; 2007.

11. Gil AC. Métodos e técnicas de pesquisa social. 6.ed. São Paulo: Atlas S.A.; 2008.

12. Denzin NK, Lincoln YS. Introdução: a disciplina e a prática da pesquisa qualitativa. In: Denzin NK, Lincoln YS. 0 planejamento da pesquisa qualitativa: teorias e abordagens. 2. ed. Porto Alegre: Artmed; 2006.

13. Bardin L. Análise de Conteúdo. Lisboa: Edições $70 ; 2009$.

14. Ministério da Saúde. Secretaria de Atenção à Saúde. Departamento de Atenção Básica. Política Nacional de Atenção Básica/ Ministério da Saúde. Secretaria de Atenção à Saúde. Departamento de Atenção Básica. - Brasília: Ministério da Saúde; 2012 [cited 2019 Abr 02]. Available from: http://189.28.128.100/dab/docs/publicacoes/geral/ pnab.pdf

15. Davini MC. Practicas laborales em los servicios de salud: las condiciones del aprendizaje. In: Educación permanente de personal de salud. Serie Desarrollo de recursos humanos, n.100. Cuernavaca. Organización Panamericana de La salud. EUA; 1994.
16. Quintana PB, Roschke MAC, Ribeiro ECO. Educação permanente processo de trabalho e qualidade de serviço na saúde. Escola de Saúde [cited 2019 Abr 02]. Available from: http://escoladesaude.pr.gov.br/ arquivos/File/textos\%20eps/educacao permanente processo de trabalho e qualidade de servico na saude.pdf.

17. Motta JIJ. Educação permanente em saúde. Revista Olho Mágico. Londrina; 2002.

18. Ministério da Saúde. Portaria GM/MS no 198/2004, de 13 de fevereiro de 2004. Institui a política nacional de educação permanente em saúde como estratégia do Sistema Único de Saúde [document on the internet]. 2005 [cited 2019 Nov 29]. Available from: http://bvsms.saude.gov.br/bvs/publicacoes/ pacto saude volume9.pdf
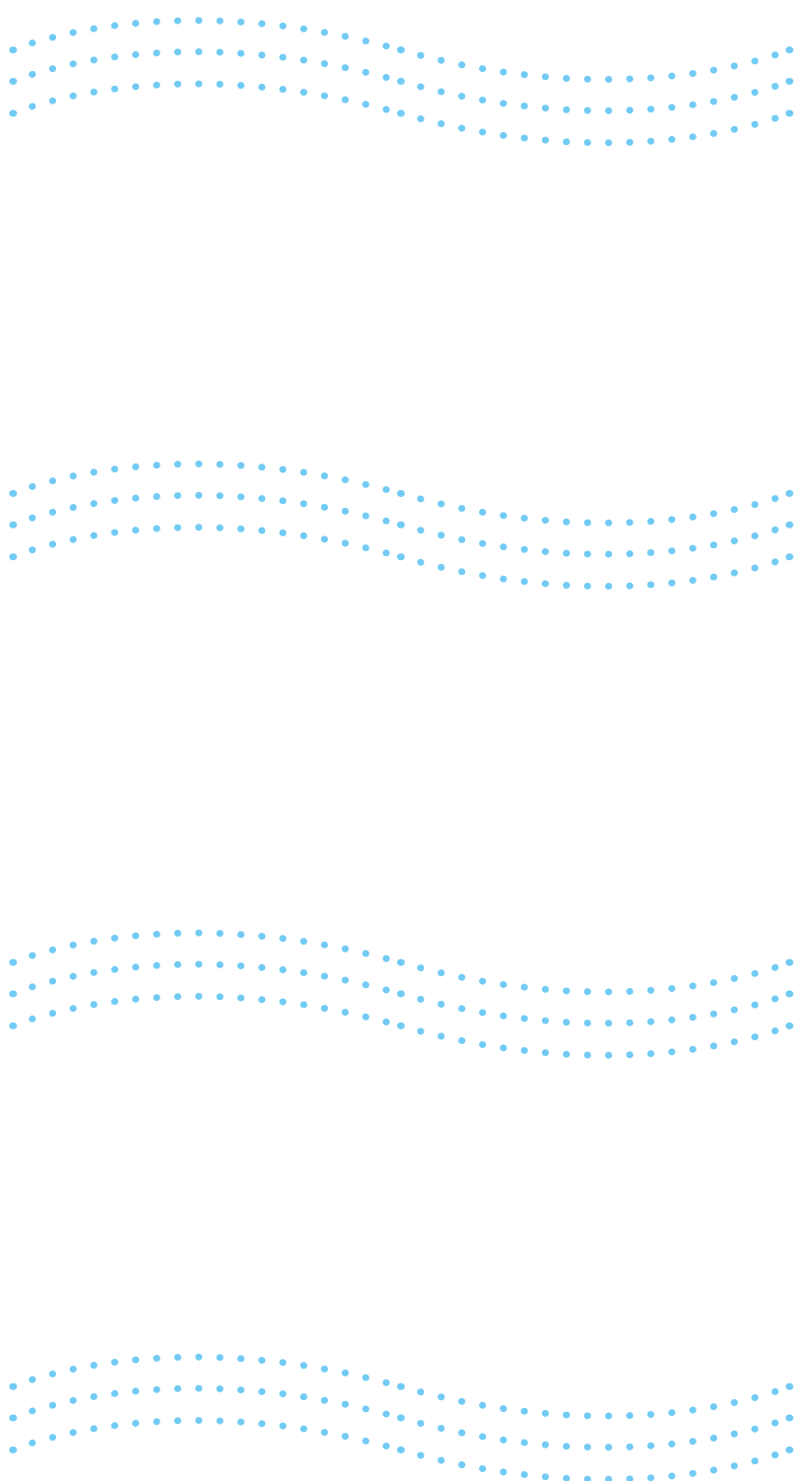